INRA Prod. Anim., 2010, 23 (2), 215-224

\title{
Les ovoproduits : des ingrédients fonctionnels pour des matrices complexes
}

\author{
M. ANTON ${ }^{1}$, F. NAU2,3, V. LECHEVALIER 2,3, C. GUÉRIN-DUBIARD ${ }^{2,3}$, T. CROGUENNEC 2,3 \\ ${ }^{1}$ INRA, UR1268 Biopolymères Interactions Assemblages, Equipe Interfaces et Systèmes Dispersés, \\ F-44316 Nantes, France \\ 2 INRA, UMR 1253 Science et Technologie du Lait et de l'Euf, \\ F-35042 Rennes, France \\ ${ }^{3}$ Agrocampus Ouest, Science et Technologie du Lait et de l'Euf, \\ F-35042 Rennes, France \\ Courriel : anton@nantes.inra.fr
}

L'œuf de poule est un ingrédient polyfonctionnel, en raison des propriétés émulsifiantes, foisonnantes, gélifiantes, épaississantes, colorantes et aromatiques de ses constituants. L'industrie des ovoproduits est relativement récente (années 70) et connaît une croissance importante. Après avoir abordé la structure et les constituants de l'oeuf, cet article décrit les différentes étapes de fabrication des ovoproduits et leurs principales applications.

L'œuf de poule est qualifié d'ingrédient polyfonctionnel car, outre sa valeur nutritionnelle, il peut remplir simultanément plusieurs fonctions technologiques dans un même produit alimentaire formulé. Ses propriétés émulsifiantes, foisonnantes, gélifiantes, épaississantes, colorantes et aromatiques en font encore à l'heure actuelle un ingrédient de base universel de la cuisine domestique et de l'agroalimentaire. Pour certaines préparations, des propriétés plus spécifiques sont recherchées, ce qui peut amener à utiliser séparément le blanc et le jaune d'œuf : le blanc d'œuf est une référence en termes de foisonnement, alors que le jaune d'œuf est l'agent émulsifiant par excellence. Au niveau industriel, le cassage des œufs pour obtenir l'ingrédient blanc ou jaune (et même «l'entier») est réalisé de façon automatisée et les produits qui en sont issus sont appelés ovoproduits. Après une revue rapide des constituants du jaune et du blanc d'œuf, cet article a pour objectif de décrire les différentes étapes de fabrication des ovoproduits et leurs principales applications.

\section{1 / Structure et constituants de l'ouf}

\section{1 / Jaune d'œuf}

Le jaune d'œuf ou vitellus, correspondant à $36 \%$ du poids de l'œuf, possède un extrait sec proche de $50 \%$, constitué principalement de lipides (environ 65\% de la matière sèche) et de protéines (33\%) (Kiosseoglou 1989, Thapon et Bourgeois 1994, Li-Chan et al 1995). Il contient également une quantité importante de minéraux, dont le fer et le phosphore. La composition générale du jaune d'œuf est présentée dans le tableau 1 . Il est à noter que les lipides sont exclusivement distribués dans des assemblages lipoprotéiques.

Du point de vue de sa structure, le jaune d'œuf est un système complexe formé d'agrégats (granules) en suspension dans un fluide clair (plasma) qui contient des lipoprotéines et des protéines. Par conséquent, le jaune peut être facilement séparé en ses deux fractions par une simple centrifugation à

Tableau 1. Répartition des constituants du jaune d'œuf de poule (d'après Powrie et Nakai 1986).

\begin{tabular}{|l|l|c|c|c|c|c|}
\hline & & $\begin{array}{c}\text { \% MS } \\
\text { du jaune }\end{array}$ & $\begin{array}{c}\text { \% lipides } \\
\text { du jaune }\end{array}$ & $\begin{array}{c}\text { \% protéines } \\
\text { du jaune }\end{array}$ & $\begin{array}{c}\text { \% en } \\
\text { lipides }\end{array}$ & $\begin{array}{c}\text { \% en } \\
\text { protéines }\end{array}$ \\
\hline Jaune & & 100 & 100 & 100 & 64 & 32 \\
& 78 & 93 & 53 & 73 & 25 \\
& LDL & 66 & 61 & 22 & 88 & 10 \\
& Livétines & 10 & - & 30 & - & 96 \\
& autres & 2 & - & 1 & - & 90 \\
& & 22 & $\mathbf{7}$ & 47 & 31 & $\mathbf{6 4}$ \\
& HDL & 16 & 6 & 35 & 24 & 75 \\
& Phosvitine & 4 & - & 11 & - & 95 \\
& LDLg & 2 & 1 & 1 & 88 & 10 \\
\hline
\end{tabular}


$10000 \mathrm{~g}$ par la méthode de McBee et Cotterill (1979).

Les granules sont des agrégats circulaires d'un diamètre de 0,3 à $2 \mu \mathrm{m}$ (Chang et al 1977) et représentent 22\% de la matière sèche du jaune. Ils sont constitués de $64 \%$ de protéines, $31 \%$ de lipides et $5 \%$ de minéraux (Dyer-Hurdon et Nnanna 1993, Anton et Gandemer 1997). Ces agrégats sont formés par des ponts phosphocalciques entre les lipoprotéines de haute densité (HDL) et la phosvitine (Burley et Cook 1961, Saari et al 1964). A faible force ionique, les agrégats de granules sont insolubles, très compacts, peu hydratés et difficilement accessibles aux enzymes (Causeret et al 1991). Lorsque la force ionique augmente (au-delà de $0,3 \mathrm{M} \mathrm{NaCl}$ ), les ponts phosphocalciques sont rompus car le sodium monovalent remplace le calcium divalent. Dans de telles conditions, la solubilité des granules dépasse les $80 \%$, et peut même être total dans un milieu à $1,71 \mathrm{M} \mathrm{NaCl}$ (Cook et Martin 1969, Anton et Gandemer 1997). L'acidification ou l'alcalinisation peuvent également provoquer la rupture des agrégats de granules en raison de l'augmentation des répulsions électrostatiques internes. Nous avons récemment établi un diagramme de phase décrivant les différents états des granules en fonction du $\mathrm{pH}$ et de la force ionique (figure 1, Sirvente et al 2007). Il apparaît que dans des conditions de solubilité totale des granules, ce sont en réalité des assemblages micellaires de 100 à $200 \mathrm{~nm}$ qui restent en suspension stable dans la phase aqueuse.

Le plasma représente $78 \%$ de la matière sèche du jaune d'œuf. Il est composé de $85 \%$ de lipoprotéines de faible densité (LDL) et de 15\% de livétines (Burley et Cook 1962, Jolivet et al 2006) (tableau 1). Il contient $73 \%$ de lipides, $25 \%$ de protéines et $2 \%$ de minéraux, ce qui représente $93 \%$ des lipides du jaune et $53 \%$ des protéines. Les lipides du plasma, tous contenus dans les LDL, sont distribués en $70 \%$ de triglycérides, $25 \%$ de phospholipides et $5 \%$ de cholestérol.

Les LDL sont des particules sphériques (30-70 $\mathrm{nm}$ de diamètre) formées d'un cœur lipidique à l'état liquide (triglycérides) entouré d'un film de phospholipides et de protéines (Cook et Martin 1969, Evans et al 1973). Les LDL sont solubles quelles que soient les conditions de $\mathrm{pH}$ et de force ionique en raison de leur faible densité $(0,982)$ et de leur taille. Il ne s'agit donc pas d'une solution vraie mais d'une dispersion colloïdale.

\section{2 / Blanc d'œuf}

Le blanc d'œuf est une solution aqueuse de protéines, de glucides et de sels minéraux représentant $60 \% \mathrm{du}$ poids total de l'œuf. Il se répartit dans l'œuf en quatre couches bien distinctes :

- la couche chalazifère $(3 \% \mathrm{p} / \mathrm{p} d u$ blanc), très ferme, qui entoure la membrane vitelline et se prolonge vers les deux extrémités de l'œuf par les chalazes ;

- le blanc liquide externe $(23 \% \mathrm{du}$ blanc) au contact des membranes coquillières ;

- le blanc épais (57\% du blanc) fixé aux deux extrémités de l'œuf ;

- le blanc liquide interne $(17 \% \mathrm{du}$ blanc) entourant le jaune.

Le blanc épais est quatre fois plus concentré en ovomucine que le blanc liquide, lui conférant une structure gélifiée et une viscosité très supérieure. Au cours du stockage des œufs, des modifications physico-chimiques se produisent, se traduisant essentiellement par une augmentation du $\mathrm{pH}$ du blanc consécutive au départ de $\mathrm{CO}_{2}$ (d'un $\mathrm{pH}$ de 7,5 au moment de la ponte à un $\mathrm{pH}$ de 9,5 après quelques jours), une liquéfaction du blanc épais et une transformation de l'ovalbumine en S-ovalbumine, plus thermostable (Kato et al 1975, Yamasaki et al 2003).

Figure 1. Effet de la combinaison pH/force ionique sur la solubilisation et la nature des agrégats de granules (d'après Sirvente et al 2007).

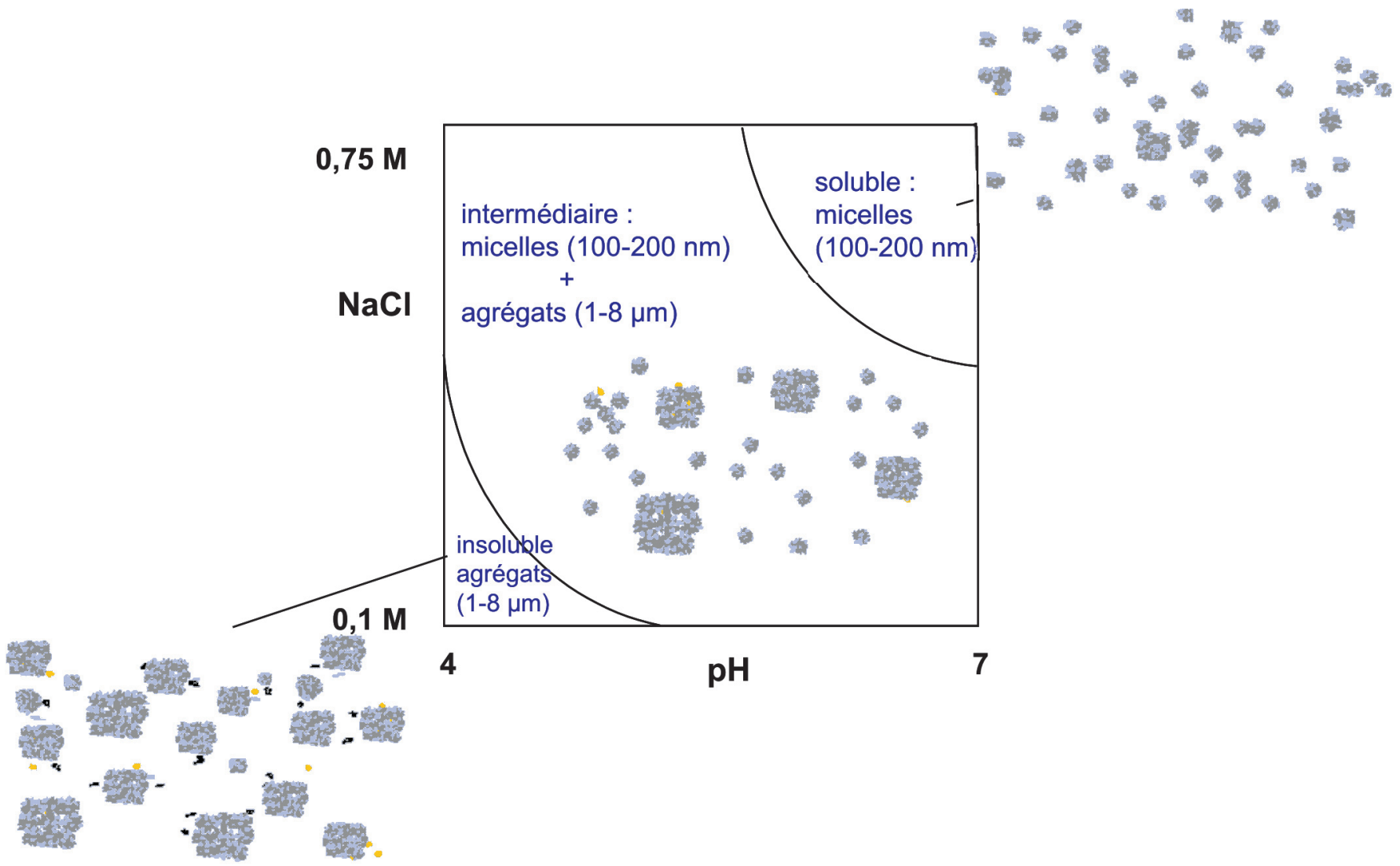


Tableau 2. Composition protéique du blanc d'œuf (d'après Li-Chan et Nakai 1989, Stevens 1991, Guérin-Dubiard et al 2006). En italique sont indiquées les protéines mineures récemment identifiées .

\begin{tabular}{|l|c|c|c|l|}
\hline Protéine & $\%$ & $\mathbf{M}(\mathbf{k D a})$ & $\mathbf{p H}_{\mathbf{i}}$ & Caractéristiques biologiques importantes \\
\hline Ovalbumine & 54 & 45 & 5 & Phosphoprotéine immunogène \\
Ovalbumine $Y$ & 5 & 44 & 5,2 & nd (non déterminé) \\
Ovalbumine $X$ & 0,5 & 56 & 6,5 & nd \\
Ovotransferrine & 13 & 76 & 6,7 & Fixe le fer, activité bactériostatique \\
Ovomucoïde & 11 & 28 & 4,8 & Inhibiteur trypsique \\
Ovomucine & $1,5-3,5$ & 230 à 8300 & $4,5-5$ & Très glycosylée, inhibe l'hémaglutination virale \\
Lysozyme & 3.5 & 14,4 & 10,7 & Lyse les parois des bactéries Gram+ \\
Ovoinhibiteur & $0,1-1,5$ & 49 & 5,1 & Inhibiteur des serine protéases \\
Ovoglycoprotéine & $0,5-1$ & 24,4 & 3,9 & nd \\
Flavoprotéine & 0,8 & 32 & 4 & Fixe la riboflavine (vitamine B2) \\
Ovostatine & 0,5 & 760 à 900 & 4,6 & Inhibiteur des serine protéases, très allergénique \\
Cystatine & 0,05 & 12,7 & 5,1 & Inhibiteur des cystéines protéases \\
Avidine & 0,05 & 68,3 & 10 & Fixe la biotine \\
Ex-FABP & $n d$ & 18 & 5,5 & Famille des lipocalines \\
Cal gamma & $n d$ & 20.8 & 6 & Famille des lipocalines \\
TENP & nd & 47,4 & 5,6 & Famille des BPI (bactericidal permeability- \\
Hep 21 & nd & 18 & 6,4 & Famille uPar/Ly6/Snake neurotoxin \\
\hline
\end{tabular}

Les protéines constituent l'essentiel de la matière sèche du blanc d'œuf avec un rapport «matière azotée/extrait sec» supérieur à $90 \%$. Le nombre total des protéines du blanc d'œuf n'est pas connu précisément. Jusqu'à très récemment en effet, seules les protéines majeures étaient identifiées. Mais le développement récent de techniques séparatives et analytiques puissantes a permis de mettre en évidence de nombreuses protéines mineures, dont certaines ont d'ores et déjà été identifiées. Les caractéristiques principales des protéines actuellement connues sont indiquées dans le tableau 2 (d'après LiChan et Nakai 1989, Stevens 1991, Guérin-Dubiard et al 2006, Mann 2007).

Les protéines du blanc d'œuf sont principalement globulaires et acides ou neutres, à l'exception du lysozyme et de l'avidine qui sont basiques. Elles sont toutes glycosylées, sauf la cystatine et la forme majeure du lysozyme. La plupart d'entre elles se dénaturent à des températures relativement basses, ainsi que par contact aux interfaces ; ces propriétés sont à l'origine des difficultés rencontrées au cours des traitements technologiques appliqués au blanc d'œuf (traitements thermiques notamment), mais elles sont aussi à l'origine de ses propriétés gélifiantes et moussantes.

La protéine majeure du blanc est l'ovalbumine (plus de $50 \%$ des protéines totales) ; cette protéine globulaire possède des groupes thiols libres et est phosphorylée. L'ovotransferrine (13\% des protéines totales) consiste en deux lobes permettant la fixation des métaux et est la protéine la plus thermosensible du blanc. L'ovomucoïde (11\% des protéines) est la plus glycosylée $(25 \%$ de sucres) et est la protéine la plus allergénique du blanc d'œuf. L'ovomucine (1 à $3,5 \%$ des protéines) a un poids moléculaire très élevé $\left(10^{4} \mathrm{kDa}\right)$ et peut s'associer, via des liaisons de nature électrostatique, avec d'autres protéines du blanc d'œuf (ovalbumine, ovotransferrine et surtout lysozyme). Enfin le lysozyme (3,5\% des protéines) est une petite protéine globulaire et basique, possédant une structure très rigide.

\section{2 / Propriétés fonctionnelles et utilisations industrielles}

L'œuf de poule a été qualifié par Baldwin (1986) d'ingrédient polyfonctionnel, tant il est vrai qu'il peut remplir plusieurs fonctions technologiques. Un grand nombre de préparations culinaires font ainsi appel à l'œuf entier qui exprime l'ensemble de ces propriétés. Pour d'autres préparations, des propriétés plus spécifiques sont recherchées, ce qui peut amener à utiliser séparément le jaune et le blanc d'œuf.

\section{1 / Propriétés émulsifiantes}

$\mathrm{Au}$ niveau industriel, le jaune d'œuf est incorporé dans de nombreux produits alimentaires pour ses propriétés émulsifiantes exceptionnelles et aussi parce qu'il procure aux aliments le goût et la couleur désirés (Bringe et Cheng 1995). Le jaune d'œuf est ainsi un ingrédient indispensable à la fabrication d'émulsions froides (mayonnaises, sauces salades) et chaudes (sauces béarnaise, hollandaise). Il participe à la formation et à la stabilisation des émulsions en constituant un film interfacial entre l'huile et l'eau. Ce film permet d'une part de diminuer la tension interfaciale entre l'huile et l'eau, et d'autre part de former une barrière protégeant les gouttelettes d'huile de la rupture (Anton et al 2009a).

Malgré l'utilisation intensive du jaune pour la fabrication de produits alimentaires formulés, et ce depuis l'invention de la mayonnaise il y a 3 siècles, le rôle de ses constituants majeurs est longtemps demeuré largement incompris en raison d'une composition mal connue et d'une structure complexe et difficile à étudier. Le jaune d'œuf est en effet un mélange complexe de protéines et de lipides formant des assemblages naturels à différentes échelles. Ces assemblages naturels contribuent à la nano- et à la macro-structure du jaune. Ainsi, l'élucidation des propriétés émulsifiantes ancestrales du jaune d'œuf réside dans la compréhension de ces différents niveaux de structure. L'un des enjeux est de comprendre les comportements des différents constituants aux interfaces huile-eau modèles et de faire le lien avec les systèmes émulsionnés. Les recherches entreprises ces dernières années ont largement contribué à cette compréhension et permettent actuellement de mieux maîtriser les émulsions fabriquées avec du jaune d'œuf. De façon globale, il apparaît que les LDL sont responsables des propriétés émulsifiantes remarquables de fabrication des émulsions du jaune d'œuf et que les granules interviennent plus dans la phase de stabilisation dans le temps des émulsions (Anton et al 2003, 2009b). Les LDL se présentent comme de véritables nano-émulsions naturelles dans la mesure où elles sont formées d'un cœur lipidique entouré d'une interface constituée de phospholipides et de protéines. Ainsi, elles servent de vecteur à des agents émulsifiants (phospholipides et protéines) qui ne seraient pas solubles 
Figure 2. Description schématique du mécanisme d'adsorption à l'interface air-eau des LDL du jaune d'œuf.

\begin{tabular}{|lll|}
\hline & & air \\
- ancrage par protéines & - dénaturation \\
- rupture & étalement & air-eau \\
& &
\end{tabular}

dans la phase aqueuse rencontrée dans les formules alimentaires. Pour s'adsorber aux interfaces entre l'huile et l'eau ou entre l'air et l'eau, elles sont ensuite déstructurées, permettant ainsi aux phospholipides et aux protéines formant sa surface de couvrir et de stabiliser cette nouvelle surface (Martinet et al 2002, 2003, Dauphas et al 2006, Anton et al 2009a) (figure 2).

\section{2 / Propriétés moussantes}

Les propriétés moussantes exceptionnelles du blanc d'œuf sont également à la base de recettes traditionnelles parmi lesquelles les meringues font certainement office de référence. En effet, l'extrême simplicité de leur formule (du blanc d'œuf et du sucre, éventuellement additionné d'arômes) permet au blanc d'œuf d'exprimer de manière optimale ses propriétés moussantes. Les paramètres technologiques influent sur la qualité finale de la mousse obtenue. C'est ainsi qu'il existe 3 types de meringues (meringue traditionnelle, meringue suisse et meringue italienne), selon d'une part que le blanc d'œuf est foisonné en présence ou non du sucre, et selon que cette opération est effectuée à température ambiante ou à chaud.

Il existe un grand nombre d'autres produits dans lesquels on incorpore du blanc d'œuf préalablement foisonné, qu'il s'agisse, d'une part, de formules exemptes de matières grasses («angel food cake») ou, d'autre part, de formules qui en contiennent (biscuits à la cuillère «sponge cake», soufflés). Enfin, les propriétés moussantes des constituants du blanc d'œuf sont également mises à profit dans la préparation d'une grande variété de produits pour lesquels l'étape de foisonnement est réalisée après mélange de tous les ingrédients, y compris lipidiques, ou à partir de l'œuf entier et non du seul blanc d'œuf (omelettes foisonnées, boudoirs, charcuterie foisonnée, mousses de légumes ou de poissons...). Sur de telles formules et préparations, la complexité des phéno- mènes est importante, foisonnement et émulsification intervenant simultanément, ce qui rend la maîtrise des paramètres physico-chimiques et technologiques de ces opérations très délicate. C'est pourquoi, malgré l'intérêt évident de telles problématiques, elles ne font l'objet que d'études empiriques, en raison de l'incapacité dans laquelle se trouvent les chercheurs de modéliser les systèmes et les phénomènes en jeu. L'étude et la caractérisation des propriétés foisonnantes du blanc d'œuf passent donc aujourd'hui essentiellement par la compréhension des comportements aux interfaces air-eau des constituants protéiques du blanc d'œuf, soit en solutions pures, soit en mélanges modèles.

Globalement, parmi les protéines de blanc d'œuf, c'est l'ovalbumine qui possède les meilleures propriétés moussantes (Townsend et Nakaï 1983). Mais dans un mélange constitué d'ovalbumine, d'ovotransferrine et de lysozyme, il existe une interaction coopérative avec coexistence des trois protéines à l'interface entre l'air et l'eau (Lechevalier et al 2005). Il ressort d'un grand nombre d'études que les propriétés moussantes des protéines de blanc prises isolément sont toujours très inférieures à celles du blanc d'œuf entier, ce qui confirme l'existence et l'importance de la prise en compte des interactions entre protéines à l'interface. Bien qu'il soit aujourd'hui démontré que le passage des solutions modèles aux solutions complexes n'est pas toujours possible, cette étape de compréhension et de modélisation sur systèmes modèles n'en demeure pas moins indispensable pour envisager à terme l'étude des milieux complexes.

\section{3 / Propriétés gélifiantes}

Les propriétés gélifiantes du blanc d'œuf sont utilisées dans de nombreuses applications alimentaires mettant en œuvre une étape de cuisson (charcuterie, terrines de poissons ou légumes, pâtisserie...). Le phénomène physicochimique exploité est celui de la thermo-gélification des protéines : on parle de gels protéiques thermotropiques. Le blanc d'œuf perd sa fluidité vers $60^{\circ} \mathrm{C}$, mais le maximum de fermeté est atteint au-delà de $70^{\circ} \mathrm{C}$. A l'exception de l'ovomucine et de l'ovomucoïde, toutes les protéines du blanc d'œuf coagulent à la chaleur. Elles n'ont cependant pas toutes le même comportement vis-à-vis des traitements thermiques. Leurs températures de dénaturation sont par exemple très différentes : à $\mathrm{pH} 7$ dans le blanc d'œuf, elles sont de l'ordre de 85,74 et $63^{\circ} \mathrm{C}$ pour respectivement l'ovalbumine, le lysozyme et l'ovotransferrine (Donovan et al 1975). Cette dernière est ainsi la protéine la plus thermosensible des protéines du blanc d'œuf, ce qui conduit habituellement à la considérer comme la protéine «initiatrice» de la gélification. On peut effectivement très nettement augmenter la température de coagulation du blanc d'œuf et améliorer les propriétés rhéologiques des gels obtenus par élimination sélective de l'ovotransferrine ou bien par augmentation de sa thermostabilité via la chélation d'ions métalliques $\left(\mathrm{Al}^{3+}, \mathrm{Fe}^{3+}\right)$. De même, les conditions de milieu ( $\mathrm{pH}$, force ionique) influencent la structure et les propriétés des gels obtenus en jouant sur l'équilibre des interactions hydrophobes attractives et électrostatiques répulsives (Croguennec et al 2002) (figure 3).

Le jaune d'œuf gélifie également quand il est soumis à un traitement thermique ou même à un procédé de congélation-décongélation. A un taux de matière sèche de $24 \%$, une concentration en $\mathrm{NaCl}$ de $0,17 \mathrm{M}$ et un $\mathrm{pH}$ de 6,1 , le jaune d'œuf et le plasma gélifient après un traitement thermique à $72^{\circ} \mathrm{C}$ pendant $2 \mathrm{~min} 30 \mathrm{~s}$, alors que les granules ne gélifient pas (Le Denmat et al 1999). Les constituants responsables de la gélification du jaune d'œuf sont donc des constituants du plasma, et plus particulièrement les LDL. Les autres constituants du jaune ne participent pas ou très peu à la gélification par la chaleur.

La gélification de solutions de plasma (et donc de jaune d'œuf) est favorisée à $\mathrm{pH}$ neutre et/ou forte concentration en $\mathrm{NaCl}$. Ces conditions correspondent à une forte neutralisation des charges des protéines des LDL, ce qui limite l'intensité de répulsion électrostatique à courte distance, et favorise ainsi l'agrégation entre protéines. Les temps de gélification enregistrés sont très courts, quelles que soient les conditions de $\mathrm{pH}$ et de force ionique. Ceci est propre aux processus de coagulation. Les gels sont opaques, signe de diffraction de la lumière par des particules de taille élevée (agrégats de protéines). 
Figure 3. Impact de la force ionique sur la thermogélification de l'ovalbumine : modèle mécanistique (d'après Doi 1993) et observations par microscopie électronique des gels de blanc d'œuf correspondant (Croguennec et al 2002).

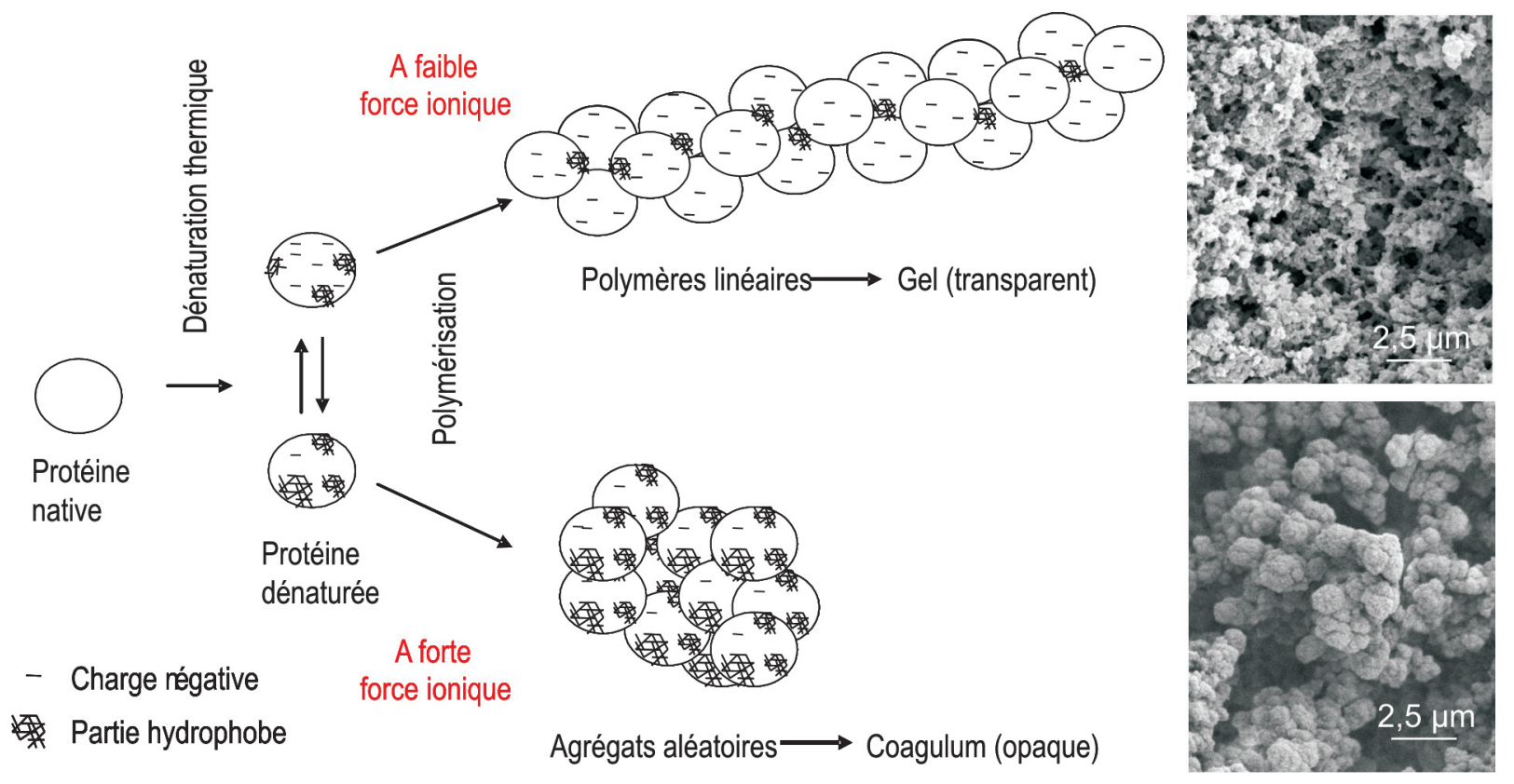

\section{3 / Fabrication des ovopro- duits}

Sont appelés ovoproduits «les produits qui ont été obtenus à partir de la transformation de l'ouf ou de ses différents composants ou mélanges, après élimination de la coquille et des membranes, ou d'une nouvelle transformation de ces produits transformés, et qui sont destinés à la consommation humaine» (arrêté du 15 avril 1992). Ils peuvent être partiellement complétés par d'autres denrées alimentaires ou additifs et ils peuvent être soit liquides, soit concentrés, séchés, cristallisés, congelés, surgelés ou coagulés. Cette définition amène à considérer deux grandes catégories d'ovoproduits : les ovoproduits dits de première transformation, correspondant à de l'œuf entier, du jaune ou du blanc d'œuf présentés sous différentes formes et destinés à être utilisés en tant qu'ingrédients techno-fonctionnels; et les ovoproduits dits de deuxième transformation qui sont en fait la reproduction à l'échelle industrielle de recettes «ménagères» classiques et qui sont donc des produits cuits ou précuits, tels que les œufs durs écalés (c'est-à-dire décoquillés), les œufs pochés, frits, les omelettes, les blancs d'œufs battus en neige, etc. L'industrie des ovoproduits offre ainsi aujourd'hui une gamme de produits, issus de schémas technologiques variés, dans le but de répondre au plus près aux utilisations techno-fonctionnelles des produits (figure 4).
L'industrie des ovoproduits, telle qu'elle existe aujourd'hui, est relativement récente (années 70 ) et connaît depuis plusieurs années une croissance importante $(+6,7 \%$ par an entre 1990 et 2000 en France). Comme toute industrie agroalimentaire, ce secteur est soumis à un certain nombre de contraintes réglementaires dont l'un des objectifs majeurs est la maîtrise de l'hygiène. Pour cela, des exigences sont édictées en matière d'organisation des sites industriels (locaux et flux) et de qualité de la matière première : seuls les œufs non incubés propres à la consommation humaine, présentant une coquille entièrement développée et sans défaut, sont utilisables pour la préparation des produits destinés à l'alimentation humaine. Le contenu d'un œuf pondu par une poule saine est stérile dans une très large majorité des cas, et si la coquille est intègre, il le restera longtemps. En revanche, dès qu'on élimine cette protection naturelle qu'est la coquille, le contenu de l'œuf est inévitablement contaminé et sa conservation pose alors de nombreuses difficultés ; or, il est impossible au niveau industriel de réaliser un cassage en conditions stériles. Si le blanc d'œuf n'est pas un milieu très favorable au développement des microorganismes, car il contient de nombreux facteurs antimicrobiens (lysozyme, ovotransferrine, ovomucoïde, avidine...), il en va tout autrement du jaune d'œuf ou de l'œuf entier qui sont des milieux de culture idéaux pour le développement des microorganismes.

Afin de maîtriser la qualité microbiologique des ovoproduits, il est donc nécessaire de limiter leur contamination initiale, d'éliminer tout ou partie de cette flore de contamination et de limiter, voire d'empêcher son développement par différents moyens de stabilisation. La difficulté à laquelle les industriels doivent alors faire face est liée à la très grande fragilité des constituants protéiques de l'œuf vis-à-vis des procédés technologiques mis en œuvre pour cette stabilisation, avec toutes les conséquences que cela induit en termes de pertes de propriétés techno-fonctionnelles, particulièrement préjudiciables dans le cas des ovoproduits de première transformation. En France, le lavage des œufs est interdit au niveau de la production et de la vente des œufs coquille, mais il est autorisé juste avant cassage (la réglementation indique d'ailleurs que «les oufs doivent si nécessaire être lavés et désinfectés»). Mais dans la pratique, très peu de fabricants d'ovoproduits français, et plus généralement européens, lavent les œufs avant cassage.

\section{1 / Cassage et séparation du blanc et du jaune}

Les œufs doivent obligatoirement être cassés individuellement, soit mécaniquement, soit manuellement. Des équipements spécifiques ont donc été développés, fonctionnant à des cadences de production pouvant aller actuellement jusqu'à 180000 œufs par heure, et permettant une séparation immédiate ou très légèrement différée dans le temps du blanc et du jaune. Les produits sont ensuite filtrés pour éliminer les fragments de coquille (objectif inférieur à 
Figure 4. Diagramme de fabrication des ovoproduits de première transformation.

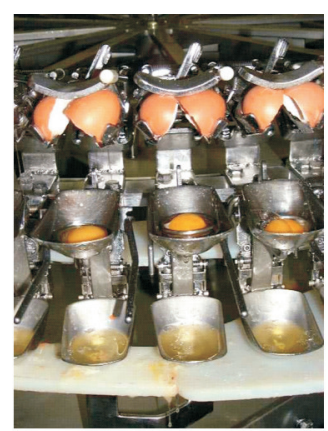

CEufs coquille<smiles>[3H][13CH3]</smiles>

nettoyage-désinfection
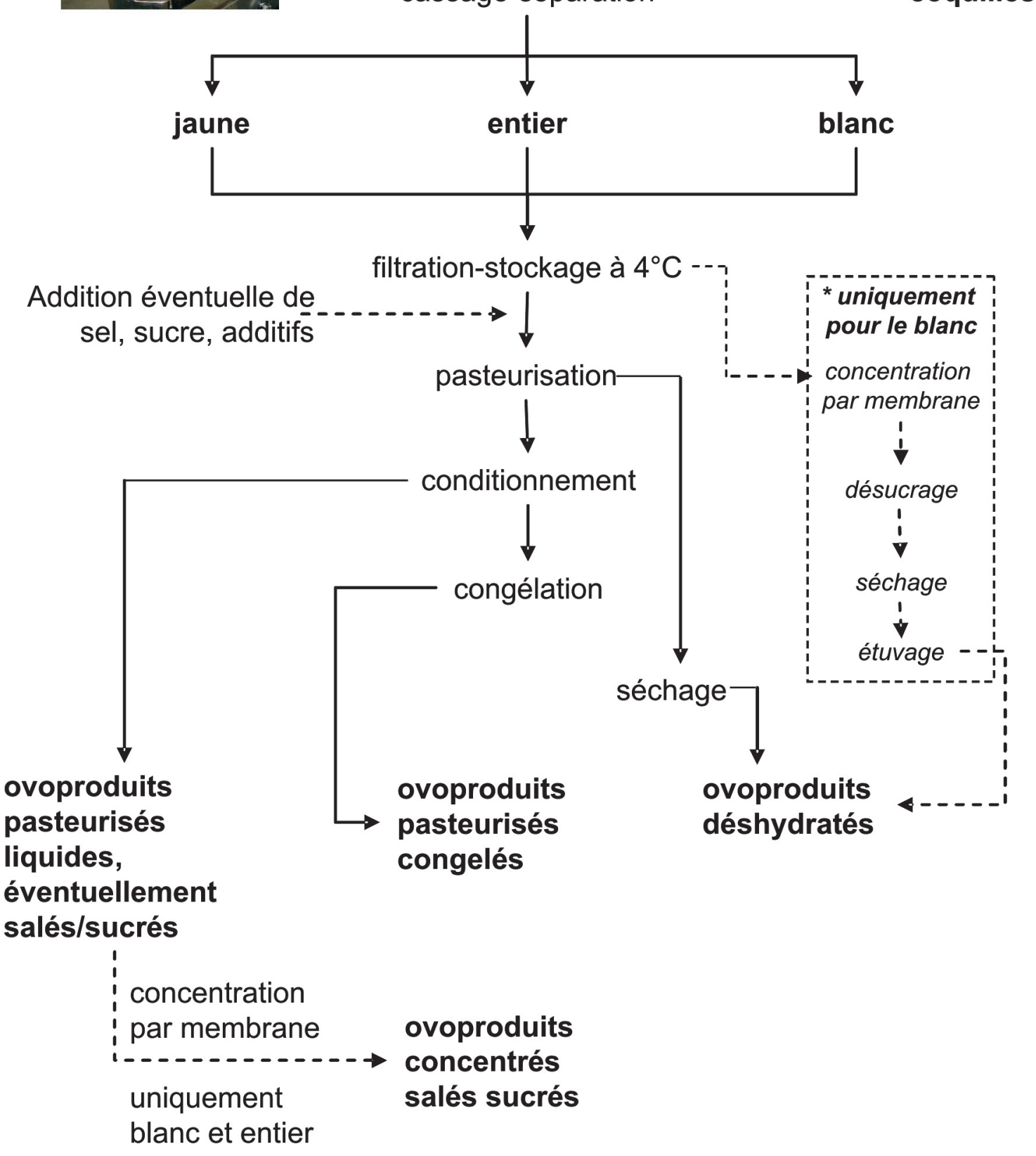

$100 \mathrm{mg}$ de coquille par $\mathrm{kg}$ de produit), puis refroidis à $4^{\circ} \mathrm{C}$ avant d'être envoyés en cuves de stockage. Des ajustements d'extrait sec, des additions de sel, de sucre ou de certains additifs autorisés (guar, xanthane dans le blanc d'œuf par exemple) peuvent être réalisés dans les cuves de stockage.
A l'issue du cassage et de la séparation, les produits bruts obtenus sont du blanc d'œuf dont l'extrait sec varie entre 10 et $11 \%$ et le $\mathrm{pH}$ entre 8,5 et 9,5 , du jaune d'œuf à $\mathrm{pH} 6,5$ et dont l'extrait sec varie de 42 à $45 \%$, et de l'œuf entier dont l'extrait sec varie de 20 à $24 \%$ et le $\mathrm{pH}$ entre 7 et 7,5. Les variations d'ex- trait sec pour le jaune et l'entier sont essentiellement liées aux performances du matériel (qualité de la séparation blanc-jaune), elles-mêmes fonction du type de matériel et de la cadence d'utilisation. Les coquilles éliminées à cette étape constituent un sous-produit avec un taux d'humidité relativement élevé 
(près de 30\%), dû aux traces de blanc d'œuf adhérentes aux coquilles. Cette humidité résiduelle est à l'origine des risques de développement microbien important dans ce sous-produit qui doit donc être stabilisé. Les coquilles sont pour cela broyées puis «essorées» par centrifugation, ce qui permet de diminuer environ de moitié le taux d'humidité. Elles peuvent ensuite être séchées, notamment (mais pas exclusivement) dans des tunnels à air chaud, avant d'être utilisées comme amendement calcaire pour l'agriculture. Une part infime de ces coquilles est également destinée à l'alimentation humaine (apport calcique), après avoir subi des traitements thermiques garantissant la destruction complète des microorganismes.

\section{2 / Décontamination et sta- bilisation des ovoproduits de 1 ère transformation}

Parmi les caractéristiques des protéines d'œuf, leur thermo-sensibilité est assurément celle qui a le plus de conséquences. Les protéines du blanc les plus sensibles à la chaleur commencent à se dénaturer dès $57^{\circ} \mathrm{C}$, ce qui signifie qu'il n'est pas possible de stériliser les ovoproduits, ni même d'appliquer des barèmes de pasteurisation tels que ceux en vigueur dans le secteur laitier par exemple. Malgré cela, les traitements de décontamination microbienne des ovoproduits les plus efficaces et les plus répandus demeurent les traitements thermiques, et parmi eux la pasteurisation.

Les traitements appliqués (couple temps-température) dépendent de plusieurs facteurs :

- de la DLC désirée : de quelques jours à moins de $4^{\circ} \mathrm{C}$ pour les ovoproduits liquides en gros conditionnement et destinés aux Industries Agro Alimentaire (IAA), à plusieurs semaines (jusqu'à 60 jours) pour des ovoproduits en petit conditionnement ( $1 \mathrm{à} 2 \mathrm{~kg}$ ) destinés aux artisans pâtissiers et cuisiniers, aux restaurants, aux petites collectivités ; des DLC de plusieurs mois à température ambiante peuvent aussi être obtenues pour des produits concentrés par des technologies à membrane (ultrafiltration) et additionnés de fortes concentrations de sucre ou de sel ;

- de la fonctionnalité désirée (propriétés moussantes ou gélifiantes du blanc d'œuf notamment), l'effet négatif des traitements thermiques sur les propriétés fonctionnelles étant proportionnel à leur sévérité.

Dans tous les cas, l'objectif à atteindre est une destruction totale des germes pathogènes et en particulier des salmonelles et la garantie de la conservation du produit jusqu'à la fin de la DLC.
Les matériels utilisés pour la pasteurisation des ovoproduits sont des échangeurs de chaleur à plaques ou tubulaires de conception classique. Les barèmes de pasteurisation habituellement mis en œuvre sont de l'ordre de $65^{\circ} \mathrm{C}$ pendant 2 à 6 minutes pour l'œuf entier et le jaune, et de l'ordre de $57^{\circ} \mathrm{C}$ pendant 2 à 6 minutes pour le blanc d'œuf. Il existe également quelques appareils spécifiques, de type tube conducteur de courant, à chauffage ohmique ou tubulaire concentrique qui permettent des traitements à plus haute température pendant un temps plus court $\left(70^{\circ} \mathrm{C}-100 \mathrm{~s}\right.$ par exemple). Ce type de traitement, suivi d'un conditionnement ultra-propre, permet l'obtention d'ovoproduits de longue conservation.

Face à l'impossibilité d'appliquer au blanc d'œuf liquide des traitements thermiques élevés, les opérateurs industriels de ce secteur ont développé depuis de très nombreuses années un procédé spécifique de traitement thermique de ce produit à l'état déshydraté. L'étuvage de la poudre de blanc d'œuf est ainsi devenu un procédé de décontamination systématique, qui offre par ailleurs l'avantage de ne nécessiter que peu d'investissements. Cette opération consiste à maintenir la poudre en chambre chaude (aux environs de $65^{\circ} \mathrm{C}$ pendant une dizaine de jours), ce qui permet d'assurer la destruction des pathogènes et d'une grande partie de la flore banale. Plus récemment, il a été montré que des barèmes d'étuvage plus sévères (de 75 à $80^{\circ} \mathrm{C}$ pendant une dizaine de jours) permettent en outre d'améliorer les propriétés fonctionnelles du blanc d'œuf (figure 5). Cette amélioration des propriétés gélifiantes et moussantes a pu être expliquée par les modifications structurales engendrées par ce type de traitement. L'augmentation de la flexibilité des protéines, l'exposition de groupements réactifs avec notamment une augmentation de l'hydrophobie de surface, ainsi que la diminution du degré d'agrégation des protéines et l'expansion de la structure des polymères formés seraient à l'origine des performances techno-fonctionnelles accrues du blanc d'œuf déshydraté étuvé (Kato et al 1989, 1990a et b).

La congélation permet d'allonger la DLC des produits jusqu'à 24 mois. Elle a cependant des conséquences importantes sur le jaune et l'entier, entraînant une gélification irréversible du produit et une modification des propriétés fonctionnelles (augmentation considérable de la viscosité du produit après décongélation). Sur le blanc, les effets sont moins importants. Cette méthode de stabilisation reste malgré tout dans la pratique de moins en moins utilisée car les temps de décongélation du produit sont relativement longs avec des risques de développement microbien élevés au cours de cette étape. Elle ne peut dans tous les cas concerner que les petits et moyens conditionnements, c'est-à-dire en-dessous de $20 \mathrm{~kg}$.

La concentration par ultrafiltration ou osmose inverse de l'œuf entier ou du blanc d'œuf, suivie d'un ajout de sucre et/ou de sel à forte concentration permet d'obtenir dans certains cas des produits stables plusieurs mois à température ambiante. La concentration d'œuf entier par un facteur 2 (soit environ $48 \%$ d'extrait sec final) associée à un ajout de sucre à $50 \%$ ou de sel à $9 \%$ permet en effet d'atteindre des $\mathrm{a}_{\mathrm{w}}$ respectivement de 0,80 et 0,85 . Dans le cas du jaune d'œuf, naturellement très concentré (extrait sec d'environ 50\%), le simple ajout de sel à $12 \%$ ou de sucre à $50 \%$ permet d'atteindre les mêmes valeurs $\mathrm{d}^{\prime} \mathrm{a}_{\mathrm{w}}$. Dans le cas du blanc d'œuf, la concentration par membrane n'est généralement effectuée que jusqu'à un extrait sec final de $33 \%$ (soit un facteur de réduction volumique de 3 ), ce qui ne permet pas ensuite d'atteindre des valeurs $\mathrm{d}^{\prime} \mathrm{a}_{\mathrm{w}}$ aussi faibles, même après ajout de sucre et/ou de sel ; malgré tout, une diminution significative de 1 ' $a_{w}$ (jusqu'à 0,88 ) peut aussi être obtenue grâce à ce procédé.

Le séchage permet quant à lui d'obtenir des ovoproduits en poudre, c'est-àdire sous la forme de conservation la plus simple, la plus longue, la plus sûre et la plus souple. Ces nombreux avantages expliquent que la fabrication des ovoproduits en poudre se développe partout dans le monde, certains pays n'utilisant plus les ovoproduits que sous cette forme. La technologie utilisée est le séchage par atomisation dans des sécheurs horizontaux ou verticaux. Dans le cas du blanc, un «désucrage» préalable est nécessaire afin d'éviter le développement de la réaction de Maillard au cours du séchage lui-même, mais surtout au cours de la phase d'étuvage décrite précédemment. Ce «désucrage» consiste à éliminer le glucose (environ 0,5 g. $1^{-1}$ dans le blanc d'œuf liquide). Il est réalisé soit par fermentation contrôlée (levures ou bactéries), soit par voie enzymatique mettant en œuvre le couple d'enzymes glucose oxydase-catalase. Cette étape de séchage et l'étuvage qui s'ensuit généralement, peuvent modifier certaines propriétés fonctionnelles des ovoproduits fréquemment dans le sens d'une amélioration (propriétés émulsifiantes, propriétés moussantes).

\section{3 / Ovoproduits cuits et/ou cui- sinés (2 ème transformation)}

Les ovoproduits de 2 ème transformation, destinés dans leur quasi-totalité à la restauration hors foyer, ne représen- 
Figure 5. Evolution des propriétés moussantes et gélifiantes au cours de l'étuvage à $67^{\circ} \mathrm{C}(\bullet)$ et $75^{\circ} \mathrm{C}(\bullet)$ de la poudre de blanc d'œuf (d'après Baron et al 2003).

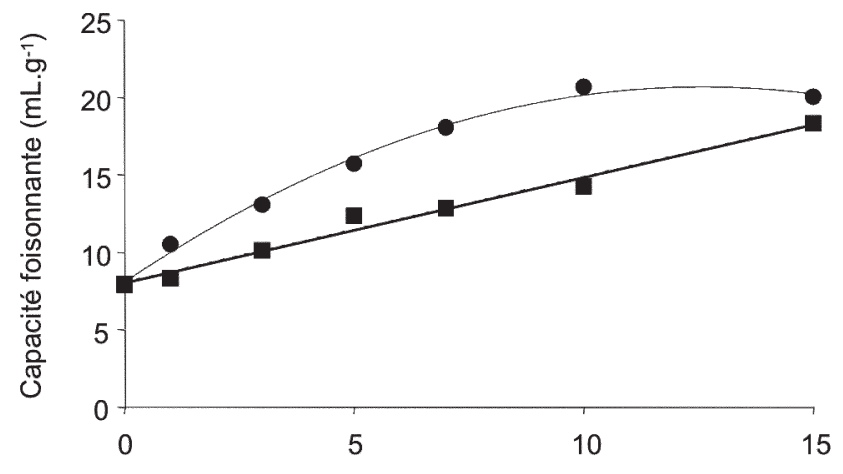

Durée de l'étuvage (jours)

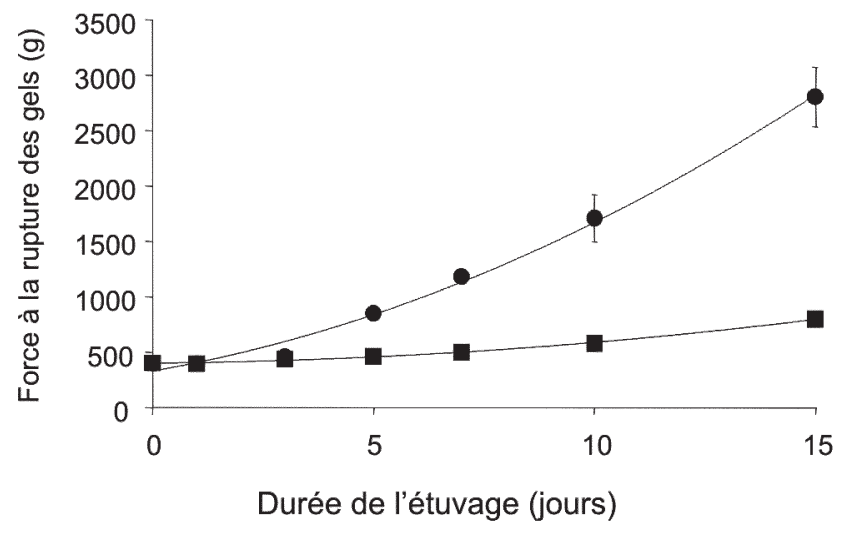

tent en France que $10 \%$ des ovoproduits totaux, et parmi eux, les œufs durs écalés représentent environ $80 \%$ du marché. Le procédé industriel pour la production des œufs écalés reprend les étapes mises en œuvre au niveau ménager. L'étape de cuisson est réalisée soit par immersion dans l'eau à $98-100^{\circ} \mathrm{C}$, soit par la vapeur. Après abaissement de la température par immersion dans un bain d'eau glacée, il faut fragmenter puis éliminer les coquilles. Cette étape est sans doute l'une des plus délicates à maîtriser à l'échelle industrielle, expliquant le très grand nombre de procédés et matériels développés et actuellement proposés par les équipementiers. La fragmentation des coquilles est généralement obtenue par des chocs provoqués entre les œufs cuits. Pour l'élimination des fragments de coquille, des systèmes à base de rouleaux, de barres, de «doigts» en caoutchouc sont utilisés, un jet d'eau sous pression venant faciliter cette opération. Dans tous les cas, un contrôle visuel et une étape manuelle d'élimination des fragments de coquille résiduels sont cependant indispensables en bout de ligne. Les œufs durs écalés sont ensuite rincés, égouttés puis conditionnés, soit en saumure, soit sous film plastique, éventuellement sous

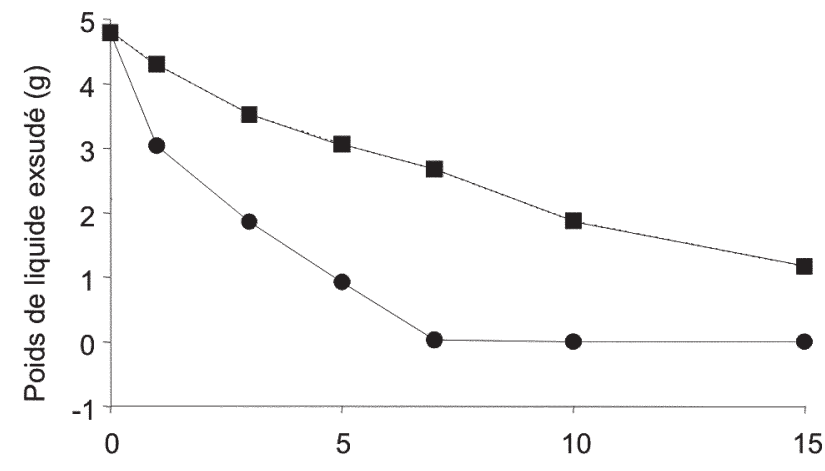

Durée de l'étuvage (jours)

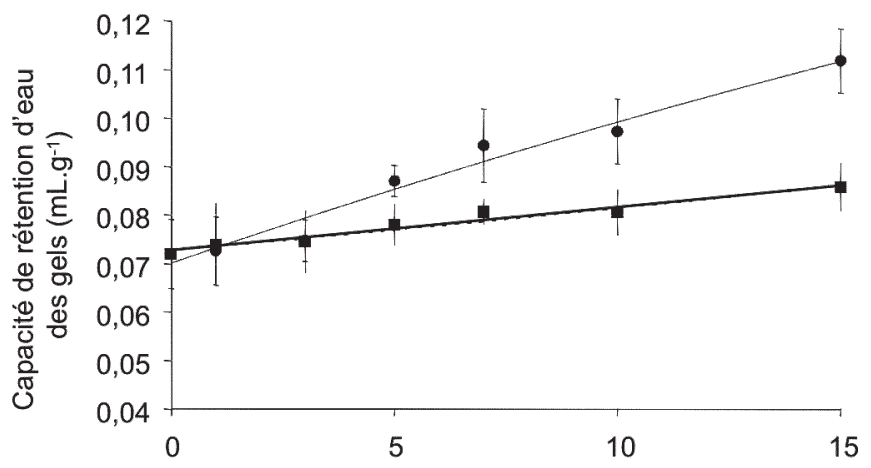

Durée de l'étuvage (jours)

atmosphère modifiée. Les deux points clés que les industriels doivent maîtriser dans le procédé de fabrication sont le niveau de cuisson de l'œuf et la qualité de l'écalage. Le niveau de cuisson doit permettre la cuisson complète du jaune d'œuf, sans toutefois atteindre une surcuisson qui risquerait d'entraîner l'apparition d'un liseré vert-noir à la surface du jaune ; ce défaut d'aspect traduit en effet la formation de sulfure de fer via une réaction chimique entre le soufre abondant dans le blanc et le fer présent dans le jaune, la cinétique de cette réaction étant totalement dépendante du couple temps/température. La qualité de l'écalage dépend quant à elle de la facilité avec laquelle les fragments de coquille sont séparés du blanc d'œuf cuit ; cette aptitude à l'écalage est clairement corrélée au $\mathrm{pH}$ du blanc d'œuf avant cuisson qui doit être supérieur à 8,5 ; ce critère exclut donc l'utilisation d'œufs très frais pour ce type de fabrication.

De nombreux procédés ont par ailleurs été développés pour produire à l'échelle industrielle des produits aussi variés que des œufs pochés, frits, brouillés, des omelettes, des blancs d'œufs en neige, etc. Malgré l'apparen- te simplicité de ces produits, de nombreuses difficultés techniques subsistent en ce qui concerne les procédés qui permettent de les fabriquer dans des conditions économiquement viables.

\section{4 / Produits d'extraction de l'œuf}

Il est possible d'extraire, à l'échelle du laboratoire ou de productions pilotes, un grand nombre de constituants de l'œuf, notamment des protéines. Même si ces produits sont pour beaucoup disponibles auprès des fournisseurs de produits biochimiques, seul le lysozyme, extrait par chromatographie d'échange de cations, est produit à l'échelle industrielle, et utilisé tant en agroalimentaire qu'en pharmacie pour ses propriétés antibactériennes. L'extraction de phospholipides pour des usages en agroalimentaire et en cosmétique est actuellement en développement.

\section{Conclusion}

L'industrie des ovoproduits est un secteur en pleine croissance qui, à l'heure actuelle, utilise essentiellement des procédés «basiques» (traitements 
thermiques, séchage) pour des produits peu élaborés, ce qui ne signifie pas pour autant que toutes les difficultés technologiques ont été levées. Toutefois, les connaissances générées ces dernières années, notamment dans les laboratoires de recherche, ont permis de mieux comprendre les fonctionnalités des consti- tuants de l'œuf; elles ont également permis de proposer des pistes de développement pour adapter les procédés utilisés, en vue de maîtriser leur impact sur ces fonctionnalités, qu'il s'agisse de minimiser la dépréciation engendrée par certains procédés ou au contraire d'amplifier les propriétés naturelles de l'oeuf. De même, les progrès réalisés dans l'extraction et la connaissance des constituants mineurs de l'œuf et de leurs propriétés biologiques ouvrent des perspectives de développement de fractions pour des applications autres que l'agroalimentaire (biologie, médecine, pharmacie).

\section{Références}

Anton M, Gandemer G, 1997. Composition, solubility and emulsifying properties of granules and plasma of hen egg yolk. J. Food Sci., 62, 484-487.

Anton M., Martinet V., Dalgalarrondo M., Beaumal V., David-Briand E., Rabesona H., 2003. Structural and chemical characterization of low-density lipoprotein purified from hen egg yolk. Food Chem., 83, 175-183.

Anton M., Belhomme C., Sirvente H., Beaumal V., David-Briand E., 2009a. What are the keypoints to understand the physicochemical and biological activities of egg compounds? 13 th Eur. Symp. Quality of Eggs and Egg Products, 21-25 juin, Turku, Finlande. 215-224.

Anton M., Nau F., Lechevalier V., 2009b. Egg proteins. In: Handbook of Hydrocolloids. Phillips G.O., Williams P.A. (Eds.). Woodhead Publishing Limited, Abington, Cambridge, UK, 2 ème Ed., 215-224.

Baldwin R.E., 1986. Functional properties of eggs in foods. In: Egg science and technology. Stadelman W.J., Cotterill O.J., The Haworth press, London, UK.

Baron F., Nau F., Guerin-Dubiard C., Gonnet F., Dubois J.J., Gautier M., 2003. Effect of dry heating on the microbiological quality, functional properties, and natural bacteriostatic ability of egg white after reconstitution. J. Food Prot., 66, 825-832.

Bringe N., Cheng J., 1995. Low-fat, lowcholesterol egg yolk in food applications. Food Technol., 49, 94-106.

Burley R.W., Cook WH., 1961. Isolation and composition of avian egg yolk granules and their constituents $\alpha$ - and $\beta$-lipovitellins. Can. J. Biochem. Physiol., 39, 1295-1307.

Burley R.W., Cook W.H., 1962. The dissociation of $\alpha$ and $\beta$ lipovitellin in aqueous solution. Part I. Effect of $\mathrm{pH}$, temperature and other factors. Can. J. Biochem. Physiol., 40, 363372 .

Causeret D., Matringe E., Lorient D., 1991. Ionic strength and $\mathrm{pH}$ effects on composition and microstructure of yolk granules. J. Food Sci., 56, 1532-1536.

Chang C.M., Powrie W.D., Fennema O., 1977. Microstructure of egg yolk. J. Food Sci., 42, 1193-1200.

Cook W.H., Martin W.G., 1969. Egg lipoproteins. In: Structural and functional aspects of lipoproteins in living systems. Tria E., Scanu A.M. (Eds). Academic Press, New York, USA 579-615.

Croguennec T., Nau F., Brulé G., 2002. Influence of $\mathrm{pH}$ and salts on egg white gelation. J. Food Sci., 67, 608-614.
Dauphas S., Beaumal V., Riaublanc A., Anton M., 2006. Hen egg yolk low density lipoproteins film spreading at the air-water and oil-water interfaces. J. Agric. Food Chem., 54, 3733-3737.

Doi E., 1993. Gels and gelling of globular proteins. Trends Food Sci. Technol., 4, 1-5.

Donovan J.W., Mapes C.J., Davis J., Garibaldi J., 1975. A differential scanning calorimetric study of the stability of egg white to heat denaturation. J. Sci. Food Chem., 26, $73-83$

Dyer-Hurdon J.N., Nnanna I.A., 1993. Cholesterol content and functionality of plasma and granules fractionated from egg yolk. J. Food Sci., 58, 1277-1281.

Evans R.J., Bauer D.H., Bandemer S.L., Vaghefi S.B., Flegel C.J., 1973. Structure of egg yolk very low density lipoprotein: Polydispersity of the very low density lipoprotein and the role of lipovitellenin in the structure. Arch. Biochem. Biophys., 154, 493-500.

Guérin-Dubiard C., Pasco M., Mollé D., Désert C., Croguennec T., Nau F., 2006. Proteomic analysis of hen egg white. J. Agric. Food Chem., 54, 3901-3910.

Jolivet P., Boulard C., Beaumal V., Chardot T., Anton M., 2006. Protein components of low-density lipoproteins purified from hen egg yolk. J. Agric. Food Chem., 54, 4424-4429.

Journal Officiel, 1992. Arrêté du 15 avril 1992 relatif aux conditions hygiéniques et sanitaires de production et de mise sur le marché des ovoproduits, J.O. du 14 juin 1992.

Kato A., Ibrahim H.R., Watanabe H., Honma K., Kobayashi K., 1989. New approach to improve the gelling and surface functional properties of dried egg white by heating in dry state. J. Agric. Food Chem., 37, 433-437.

Kato A., Imoto T., Yagishita K., 1975. The binding groups in ovomucin-lysozyme interaction. Agric. Biol. Chem, 39, 541-544.

Kato A., Ibrahim H.R., Watanabe H., Honma K. Kobayashi K., 1990a. Structural and gelling properties of dry-heated egg white proteins. J. Agric. Food Chem., 38, 32-37

Kato A., Ibrahim H.R., Watanabe H. Honma K., Kobayashi K., 1990b. Enthalpy of denaturation and surface functional properties of heated egg white proteins in the dry state. J. Food Sci., 55, 1280-1283.

Kiosseoglou V.D., 1989. Egg yolk. In: Food emulsifiers: chemistry, technology, functional properties and applications, Charalambous G., Doxastakis G. (Eds). Elsevier, London, 63-85.

Lechevalier V., Croguennec T., Pezennec S., Guérin-Dubiard C., Pasco M., Nau F., 2005.
Evidence for synergy in the denaturation at the air-water interface of ovalbumin, ovotransferrin and lysozyme in ternary mixture. Food Chem., 92, 79-87.

Le Denmat M., Anton M. Gandemer G., 1999. Protein denaturation and emulsifying properties of plasma and granules of egg yolk as related to heat treatment. J. Food Sci., 64 194-197.

Li-Chan E., Nakai S., 1989. Biochemical basis for the properties of egg white. Crit. Rev. Poult. Biol., 2, 21-58.

Li-Chan E.C.Y., Powrie W.D., Nakai S., 1995. The chemistry of eggs and egg products. In: Egg Science and Technology. Stadelman W.J., Cotterill O.J., The Haworth press, London, UK, $4^{\text {th }}$ ed., chap. 6, 105-175.

Mann K., 2007. The chicken egg white proteome. Proteomics, 7, 3558-3568.

Martinet V., Beaumal V., Dalgalarrondo M. Anton M., 2002. Emulsifying properties and adsorption behavior of egg yolk lipoproteins (LDL and HDL) in o/w emulsions. Recent Research Dev. Agric. Food Chem., 37, 103-116.

Martinet V., Saulnier P., Beaumal V., Courthaudon J.L., Anton M., 2003. Surface properties of hen egg yolk low-density lipoproteins spread at the air-water interface. Colloids Surf B: Biointerfaces, 31, 185-194.

McBee L.E., Cotterill O.J., 1979. Ionexchange chromatography and electrophoresis of egg yolk proteins. J. Food Sci., 44, 657-666.

Powrie W.D., Nakai S., 1986. The chemistry of eggs and egg products. In: Egg Science and Technology. Stadelman W.J., Cotterill O.J.

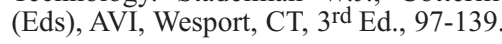

Saari A., Powrie W.D., Fennema O., 1964 Isolation and characterization of low-density lipoproteins in native egg yolk plasma. J. Food Sci., 29, 307-315.

Sirvente H., Beaumal V. Bialek L., Anton M., 2007. Structuration and functionalization of egg yolk plasma and granules solutions induced by mechanical treatments. J. Agric. Food Chem., 55, 9537-9544.

Stevens L., 1991. Mini-review: egg white proteins. Comp. Biochem. Physiol., 100, 1-9

Thapon J.L., Bourgeois C.M., 1994. L'œuf et les ovoproduits. Editions Tec et Doc Lavoisier, Paris, France, chap. 1., 84-94.

Townsend A.A., Nakai S., 1983. Relationship between hydrophobicity and foaming characteristics of food proteins. J. Food Sci., 48, 588-594.

Yamasaki M., Takahashi N., Hirose M., 2003. Crystal structure of S-ovalbumin as a non-loop-inserted thermostabilized serpin form. J. Biol. Chem., 278, 35524-35530. 


\section{Résumé}

L'œuf de poule est un ingrédient polyfonctionnel, c'est-à-dire qu'il peut remplir simultanément plusieurs fonctions technologiques dans un produit alimentaire formulé. Ses propriétés émulsifiantes, foisonnantes, gélifiantes, épaississantes, colorantes et aromatiques en font un ingrédient de base de la cuisine domestique et de l'agroalimentaire. Plus spécifiquement, le jaune d'œuf est l'agent émulsifiant par excellence, alors que le blanc d'œuf est une référence en termes de foisonnement. A l'échelle industrielle, l'œuf est transformé en ovoproduits dits de première transformation (œuf entier, jaune ou blanc, à l'état liquide, congelé ou poudre), ou dans certains cas cuit et/ou cuisiné, de manière à reproduire certaines recettes ménagères classiques ; on parle alors d'ovoproduits de deuxième transformation. Cette industrie relativement récente connaît depuis plusieurs années une croissance importante. Les connaissances sur les constituants, la structure de l'ouf et la compréhension des mécanismes mis en jeu au cours des traitements thermomécaniques, issues de la recherche développée depuis plus d'une vingtaine d'années, ont permis d'une part de mieux maîtriser les fonctionnalités des constituants de l'œuf et d'autre part d'adapter les procédés de transformation utilisés dans le secteur des ovoproduits. De même, les progrès réalisés dans l'extraction et la connaissance des constituants mineurs de l'œuf et de leurs propriétés biologiques ouvrent des perspectives de développement pour des applications hors du secteur de l'agroalimentaire (biologie, médecine, pharmacie). Cet article, a pour objectif de décrire les différentes étapes de fabrication des ovoproduits, après avoir abordé succinctement la structure, les constituants, et les principales applications de l'œuf et de certains de ses constituants.

\section{Abstract}

\section{Egg products: functional ingredients for complex matrices}

Hen egg is a polyfunctional ingredient as it can bring concurrently different functionalities in formulated foods. Its emulsifying, foaming, gelling, thickening, colouring and aromatising properties are largely researched. At industrial scale, egg is transformed in egg products of first (whole egg, yolk, albumen) or second (classical recipes) transformation. This industry is rather recent and is knowing a continuous growth. During the last fifteen years, laboratory researches have brought important knowledge concerning constituents and different levels of egg structure, understanding of functional mechanisms and thermomechanical treatment impact. These knowledges serve now to better control functionalities of egg products in food applications. Furthermore, advances realised on extraction of minor constituents could serve for new applications in biological and pharmaceutical industry. The aim of this paper is to describe the different steps to obtain egg products, with a brief presentation of structure, constituents and functionalities of egg.

ANTON M., NAU F., LECHEVALIER V., GUÉRIN-DUBIARD C., CROGUENNEC T., 2010. Les ovoproduits : des ingrédients fonctionnels pour des matrices complexes. In : Numéro Spécial, Qualité de l'œuf. Nys Y. (Ed). Inra Prod. Anim., 23, $215-224$

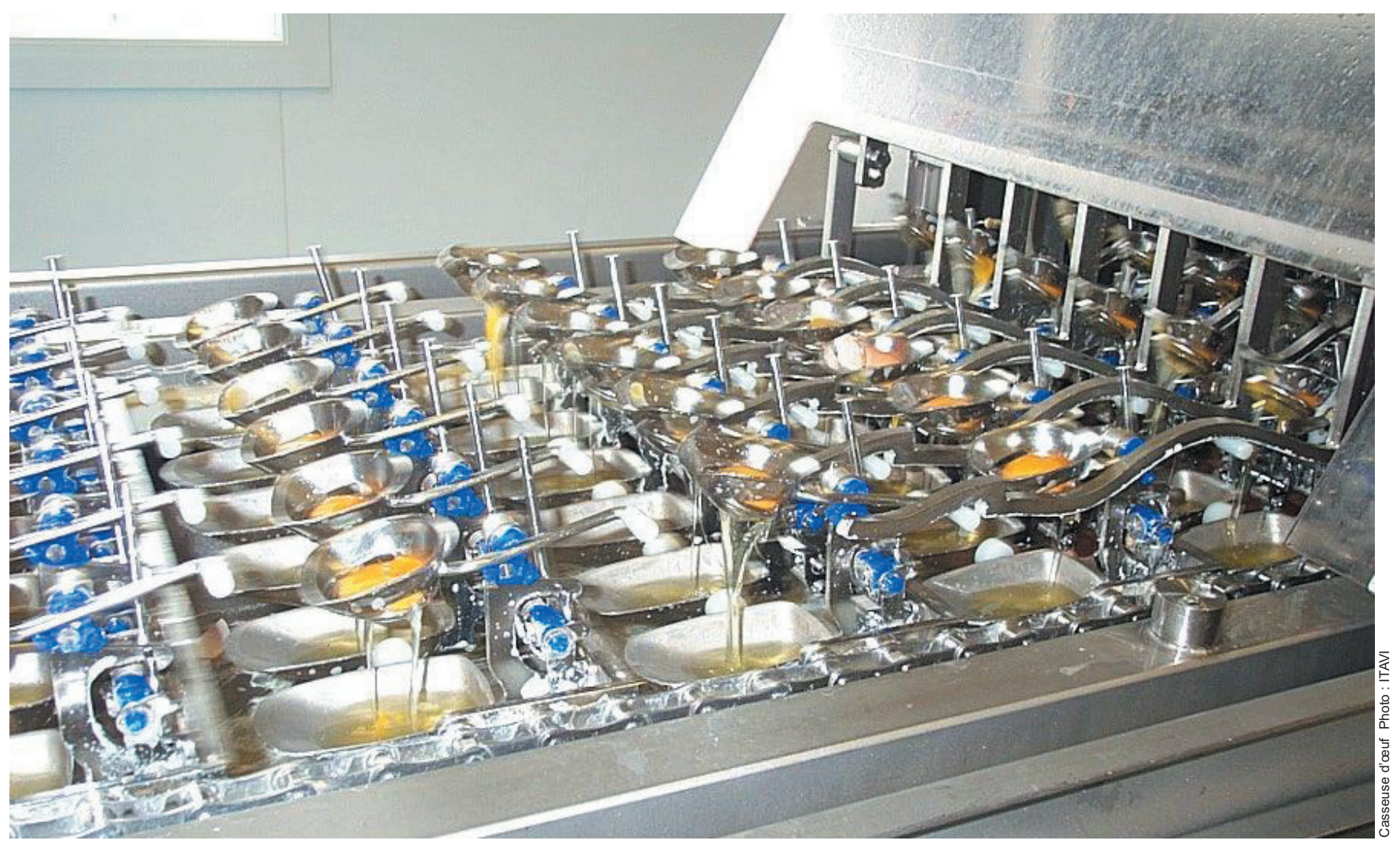

